\title{
Rare Ovarian Tumours
}

\section{NAVDEEP SINGH}

Common things are common. But rare tumours are fascinating and eye openers. It is exciting to diagnose and treat rare ovarian tumours. Literature is flooded with such tumours. Among hematological malignancies, rare ovarian tumours include primary ovarian granulocytic sarcoma, primary ovarian precursor B cell lymphoblastic lymphoma, primary extranodal non-hodgkins lymphoma and primary ovarian Hodgkins lymphoma. Primary primitive neuroectodermal tumour (PNET) ovary, primary ovarian melanoma, clear cell ovarian tumour and sex cord stromal tumour constitute other rare solid ovarian tumours.

Numerous case reports of primary granulocytic sarcomas are available all over the world including India. These are rare extramedullary tumours of malignant myeloid precursor cells. Primary involvement of ovary is exceedingly rare which usually leads to underdiagnosis. A high index of suspicion aided by immunohistochemistry and staining with myeloperoxidase establishes the correct diagnosis. These tumours are treated with standard $3+7$ induction and consolidation chemotherapy.

Precursor B cell lymphoblastic lymphoma of the ovary is an extremely rare tumour with only four cases reported so far. Primary ovarian lymphomas are mostly diffuse large B cell type, though case reports of $\mathrm{T}$ cell and Burkitts lymphoma do exist. Their cell of origin is an enigma as lymphoid aggregates are not present in normal ovarian tissue. Small, round blue morphology with LCA positivity clinches the diagnosis. These tumours are treated with standard R-
CHOP chemotherapy. A single case report of primary ovarian Hodgkins lymphoma has been reported. This is really amazing and stunning.

Apart from lymphomas, PNET of ovary constitute less than $1 \%$ cases. These tumours are MIC-2 positive. About 30 such cases have been reported so far. These are treated with surgery and combination chemotherapy. Few case reports of primary ovarian osteosarcoma and primary ovarian melanomas have reported. These are exceedingly rare ovarian tumours.

Sex cord stromal tumours account $7 \%$ of all malignant ovarian neoplasm that develop from the sex cords and ovarian stroma. These are detected typically at an early stage, and may recur as late as 30 years after initial treatment. Adult granulosa cell tumour is most common type. Surgery is the cornerstone of treatment for both primary and recurrent disease. There is no data to support any kind of post operative adjuvant treatment for stage I sex cord tumours. Platinum based chemotherapy is usually used for patients with advanced stage or recurrent disease.

Clear cell carcinoma of ovary accounts for $5-6 \%$ of ovarian malignancies. It has unique clinical features such as high incidence of stage I disease, a large pelvic mass, association with thromoembolic phenomena, hypercalcemia and endometrosis. It is resistant to platinum based chemotherapy. Surgery is the major modality of treatment.

Times to come will unfold many other rare ovarian tumours. 